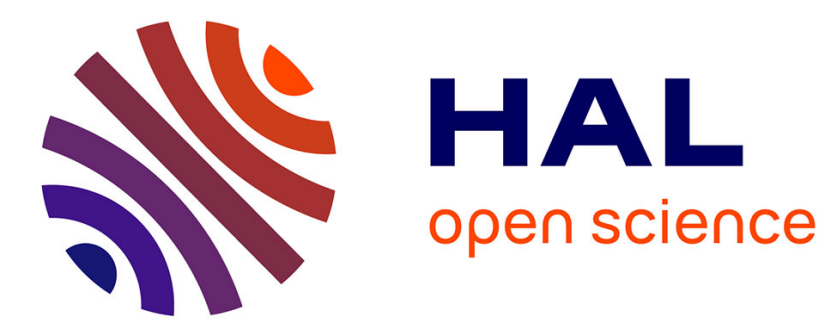

\title{
A numerical Eulerian approach to mixing by chaotic advection
}

Valérie Toussaint, Philippe Carrière, Florence Raynal

\section{To cite this version:}

Valérie Toussaint, Philippe Carrière, Florence Raynal. A numerical Eulerian approach to mixing by chaotic advection. Physics of Fluids, 1995, 7 (11), pp.2587-2600. 10.1063/1.868707 . hal-01348398

\section{HAL Id: hal-01348398 \\ https://hal.science/hal-01348398}

Submitted on 22 Jul 2016

HAL is a multi-disciplinary open access archive for the deposit and dissemination of scientific research documents, whether they are published or not. The documents may come from teaching and research institutions in France or abroad, or from public or private research centers.
L'archive ouverte pluridisciplinaire HAL, est destinée au dépôt et à la diffusion de documents scientifiques de niveau recherche, publiés ou non, émanant des établissements d'enseignement et de recherche français ou étrangers, des laboratoires publics ou privés. 


\title{
A numerical Eulerian approach to mixing by chaotic advection
}

\author{
Valérie Toussaint, Philippe Carrière, and Florence Raynal \\ Laboratoire de Mécanique des Fluides et d'Acoustique, URA CNRS 263, \\ Ecole Centrale de Lyon-Université Claude Bernard Lyon I, BP 163-69131 Ecully, Cedex, France
}

(Received 22 March 1995; accepted 18 July 1995)

\begin{abstract}
Results of numerical simulation of the advection-diffusion equation at large Péclet number are reported, describing the mixing of a scalar field under the action of diffusion and of a class of steady, bounded, three-dimensional flows, which can have chaotic streamlines. The time evolution of the variance of scalar field is calculated for different flow parameters and shown to undergo modulated exponential decay, with a decay rate which is a maximum for certain values of the flow parameters, corresponding to cases in which the streamlines are chaotic everywhere. If such global chaos is present, the decay rate tends to oscillate, whereas the presence of regular regions produces a more constant decay rate. Significantly different decay rates are obtained depending on the detailed properties of the chaotic streamlines. The relationship between the decay rate and the characteristic Lyapunov exponents of the flow is also investigated. (C) 1995 American Institute of Physics.
\end{abstract}

\section{INTRODUCTION}

Flows exhibiting chaotic streamlines (particle trajectories for time dependent flows) have been much studied these last ten years (see Ottino, ${ }^{1}$ and the special issue of Phys. Fluids, ${ }^{2}$ for review on the subject). As pointed out by Aref, ${ }^{3}$ a particularly interesting feature is that the intrinsic dispersion properties provide an efficient method for the mixing of scalar fields, even using simple velocity fields (laminar flows, for instance). Indeed, if turbulent flows, which produce random motion of particles in the fluid, are mainly used in classical mixing systems, they are not always very convenient, either because the viscosity of the fluid is very high or because they lead to undesirable side effects on the substance to mix. Several two-dimensional, time-periodic flow fields generating chaotic advection have been proposed in the literature, the journal bearing flow (Aref and Balachandar, ${ }^{4}$ Chaiken et al. ${ }^{5}$ Chaiken et al. $\left.{ }^{6}\right)$ or a cavity with moving side walls (Chien et $^{\mathrm{al}} .^{7}$ ) for instance. For two-dimensional flows, the velocity field must be time dependent in order to produce chaotic advection. In three dimensions, the situation is different since steady three-dimensional flows may exhibit chaotic streamlines: Hénon ${ }^{8}$ and Dombre et al. ${ }^{9}$ studied the Arnold-Beltrami-Childress (ABC) flow as a prototypical example for inviscid fluids. Such behavior is not restricted to open flows: Bajer and Moffatt ${ }^{10}$ proposed a class of bounded flows exhibiting chaotic streamlines.

For these flows, stirring efficiency is usually estimated from the size of the chaotic region of the flow, evaluated using Poincaré sections. Optimal stirring requires that the chaotic region covers a large part of the fluid domain. Since the particle trajectories (or streamlines for steady flows) are highly nonintegrable, numerical computation is required to determine these chaotic regions. Note however, that analytical predictions may be available using the tangle dynamic approach: Kaper and Wiggins ${ }^{11}$ successfully applied this method to the large scale chaos appearing in a journal bearing flow. Further considerations on stirring efficiency yield the stretching efficiency of material interfaces in the chaotic region: to first order, the stretching rate is often approximated by the positive Lyapunov exponent, ${ }^{12,13}$ although this is only an asymptotic limit valid for infinite times. This assumption will be considered here for comparison with our numerical simulations, sce section $\mathrm{V}$.

The mixing process also requires the molecular diffusion which determines the homogenization of the smallest scales of the scalar field. Indeed, the physical mechanism of mixing may be thought as a competition between the action of convection, which creates smaller and smaller length scales of the scalar field by successive stretching and folding, and the action of diffusion, which homogenizes the local disparities of this scalar field. To take into account molecular diffusion, a Lagrangian approach may be pursued by adding terms of Brownian motion type to the classical equation for trajectories, leading to a generalized Langevin equation. Such an approach was used by Aref and Jones ${ }^{12}$ (see also Jones ${ }^{13}$ ) and allowed comparison with a scaling theory of the asymptotic dependence on the molecular diffusivity. The main limitation of the Lagrangian approach is due to its statistical nature which requires the computation of a large number of particle trajectories, and global characterization of the scalar field at large time is rather difficult to obtain. Similarly, the alternative Eulerian approach is limited, in many cases, by the presence of small scales of the scalar field which must be accurately calculated since they reflect the mechanism of mixing by chaotic advection. Nevertheless; there may be some configurations for which such an approach is available, the downside being that they represent model rather than physically relevant flow fields. The simulation of the Eulerian advection-diffusion equation allows one direct access to the local and global characteristics of the scalar field, as well as to its gradients which play an important role in the mixing process. In particular, it is possible to study the long time evolution of the scalar field as well as the influence of streamline topology on global mixing efficiency.

The present paper describes numerical simulation of the mixing of a diffusive scalar field under the action of a steady, bounded, three-dimensional flow exhibiting chaotic streamlines, at large but finite Péclet number $\left(10^{4}-10^{5}\right)$. For such large values, the length scale at which diffusion becomes important is sufficiently small compared to the overall geom- 
etry so that the action of chaotic advection is clearly significant. In this way, asymptotic limiting tendencies become plainly visible, whereas too small a Péclet number would result in specific evolution characteristics, see section IV. Owing to the presence of small scales of the scalar field, numerical simulation of the Eulerian advection-diffusion equation is highly computer intensive and, for this reason, the problem considered must be chosen to make the computation as efficient as possible. Indeed, the choice of configuration was mainly determined by computational considerations. $\Lambda$ parallel piped domain allowing a simple spectral method and an analytical expression of the velocity field were considered to be of particular interest. The additional computing cost resulting from the three-dimensionality of the problem was partly compensated by the fact that most of the calculations can be performed as two independent twodimensional ones. The typical computing time for most of the runs we performed was less than one hour on a CRAY YMP which enabled a number of different experiments. However, for the smallest diffusivity considered in the paper, the computing time was about eight hours. Another interesting feature, since we deal with finite Schmidt numbers, is that, for steady three-dimensional chaotic advection, the topology of the streamlines is independent of time, so that there is no interaction with the characteristic time scale of diffusion. Moreover, since the advection-diffusion operator is time independent, the formal mathematical problem can be, in principle, simply expressed in terms of the spectrum of a linear elliptic opcrator with constant cocfficients (note that, in the case of a two-dimensional, time-periodic flow field the Floquet theory also leads to a similar formalism). This leads to a particularly simple theory of the global evolution of passive scalar fields and of the problem of the mixing efficiency of a prescribed flow field. However, owing to the size of the discrete operator, it was not possible to solve the resulting eigenvalues problem: nevertheless, the approach used is somewhat similar to a power method for calculating the dominant eigenvalues.

Section II describes the basis of the method, the geometry and the numerical approach. Characterization of the model flow, using the classical notions of Poincare sections and Lyapunov exponents, is presented in section III. Section IV is devoted to the results of the numerical simulation of the advection-diffusion equation for different flow parameters and streamline topologies. An attempt to explain the relationship between the numerical mixing time and the computed Lyapunov exponents is presented in section V. Finally, section VI draws the main conclusions of the work.

\section{FORMULATION}

The mixing of a passive scalar field inside a bounded domain $\Omega$ having boundary $\Gamma$ is considered. The evolution of the nondimensional scalar field $c(\mathbf{x}, t)$, satisfying the initial condition:

$$
c(\mathbf{x}, 0)=c_{n}(\mathbf{x}),
$$

is governed by the nondimensional advection-diffusion equation (see Appendix A):

$$
\frac{\partial c}{\partial t}+\mathbf{U}(\mathbf{x}) \cdot \nabla c=\frac{1}{P e} \nabla^{2} c \text { in } \Omega,
$$

where $P e=R e S c$ is the Péclet number, and $\mathbf{U}(\mathbf{x})$ is a prescribed, time independent, incompressible velocity field satisfying the boundary condition:

$$
\mathbf{U} \cdot \mathbf{n}=0 \text { on } \Gamma \text {, }
$$

$\mathbf{n}$ being the outward normal to $\Gamma$. The boundary condition for $c(\mathbf{x}, t)$, that there be vanishing scalar flux across $\Gamma$, is given by:

$$
\nabla c \cdot \mathbf{n}=0 \text { on } \Gamma \text {. }
$$

Since the domain is closed, the mean value of the scalar field remains constant with time (see Appendix A). Thus, in what follows, we subtract a constant from the scalar field so that:

$$
\int_{\Omega} c d^{3} \mathbf{x}=\int_{\Omega} c_{0} d^{3} \mathbf{x}=0
$$

$c(\mathbf{x}, t)$ then represents the deviation from the perfectly mixed case in which the scalar field is zero everywhere. To quantify the mixing state in $\Omega$ at time $t$, we define the root mean square value of the scalar field:

$$
\tilde{c}(t)=\left(\int_{\Omega} c^{2} d^{3} \mathbf{x}\right)^{1 / 2},
$$

where the nondimensional volume of $\Omega$ is chosen to be unity. For a closed domain without sources of $c(\mathbf{x}, t)$, this quantity must decrease continuously with time owing to the diffusion mechanism. Indeed, from equation (2) and boundary conditions (3) and (4) we have (see Appendix A):

$$
\frac{d}{d t} \tilde{c}(t)^{2}=-\frac{2}{P e} \int_{\Omega}|\nabla c|^{2} d^{3} \mathbf{x} .
$$

Since the right hand side term of (7) is always negative, $\tilde{c}(t)$ is decreasing and it can be shown that:

$$
\tilde{c}(t) \rightarrow 0 \text { as } t \rightarrow \infty \text {. }
$$

Owing to linearity of the problem, it is always possible to scale appropriately $c(\mathbf{x}, t)$ and we may therefore assume that the initial condition satisfies:

$$
\int_{\Omega} c_{0}^{2} d^{3} \mathbf{x}=1
$$

The efficiency of the mixing mechanism is measured by the rate of decay of $\tilde{c}(t)$ with time for any initial condition $c_{0}(\mathbf{x})$ satisfying condition (9).

From a mathematical viewpoint, the problem may be expressed in terms of the spectrum of the linear elliptic operator $\left(-\mathbf{U}(\mathbf{x}) \cdot \nabla+P e^{-1} \nabla^{2}\right)$; it is known that, under certain smoothness assumptions on $\Gamma$ and $\mathbf{U}(\mathbf{x})$, this operator possesses a countable set of complex eigenvalues $\nu_{1}, \nu_{2}, \ldots$, of finite multiplicity, for which the real parts $\mathfrak{R}\left(\nu_{k}\right)$ are negative and tend to $-\infty$ when $k \rightarrow \infty$ (see, for instance, Ladyzenskaya and Ural'ceva ${ }^{14}$ ). In other words, given arbitrary initial conditions, for sufficiently long limes, we expect exponential decay of $\tilde{c}(t)$ at a rate corresponding to the real part of the 
largest eigenvalues. The main objective of this paper is to analyze the time evolution of $\tilde{c}(t)$ for various streamlines topologies and to relate its mean rate of decay to the properties of the flow field.

As already stated, we consider typical values of the Péclet number of order $10^{4}-10^{5}$. Of course, this may either result from a large Reynolds number of the flow or from a large Schmidt number of the scalar; here, since we deal with time independent velocity fields, the Reynolds number of the flow is necessarily not too large, say, at most of order unity. In any case, the velocity fields which are used, which are examples of velocity fields in the Stokes limit, are only valid for creeping flows when the Reynolds number is small. Thus, the large value of the Péclet number arises from a large value of the Schmidt number. This is, for instance, typical of fluorescent contaminants in liquids for which the Schmidt number ranges from about 2000 in water up to $10^{12}$ in glycerine. $^{15}$

Such large values of the Péclet number can give rise to numerical difficulties: from equation (7), the rate of decay of $\tilde{c}(t)$ is directly related to the mean square value of the gradient of $c$. The effect of convection is to produce high local gradients by creating small scales of the scalar field which diffuse more rapidly than the larger ones. Consequently, the ability of a numerical scheme to simulate mixing is essentially related to its ability to accurately describe the smallest scales of the scalar field, as is often the case for convective problems; turbulence modelling, for instance.

In order to avoid any problem of numerical accuracy, we undertake first investigations in a simple geometry for which a spectral method is available, as is often the case for direct numerical simulation of turbulence. The domain $\Omega$ considered here is thus simply a unit cube: the origin of the Cartesian coordinates is taken to be at a corner of the cube, with the three axes parallel to the edges. Owing to the boundary conditions (4), it is possible to expand the scalar field using a basis of cosine functions in each direction, which are eigenfunctions of the diffusion operator alone. Note that, since the fluid domain is bounded, sine functions may be avoided and that the computing cost is lower than for a full expansion. Introducing this expansion in equation (2) and applying a standard Galerkin method, one obtains a set of coupled linear ordinary differential equations for the amplitude of each mode. Since analytical forms of the velocity fields are used, it is possible to calculate exact expressions for the coefficients. This set of equations is then integrated in time using an explicit fourth order Runge-Kutta scheme.

\section{THE VELOCITY FIELDS}

The work of Bajer and Moffatt ${ }^{10}$ suggests that chaotic streamlines are usual rather than occasional results of steady three dimensional laminar flows in confined geometry. However, only a few such velocity fields lead to analytical expressions. Owing to the cubic geometry, we propose to analyze the velocity field due to the superposition of two twodimensional recirculating flows, whose axes of rotation are oriented orthogonal to each other (see Fig. 1).

The velocity field $\mathbf{U}_{1}$ is chosen as:
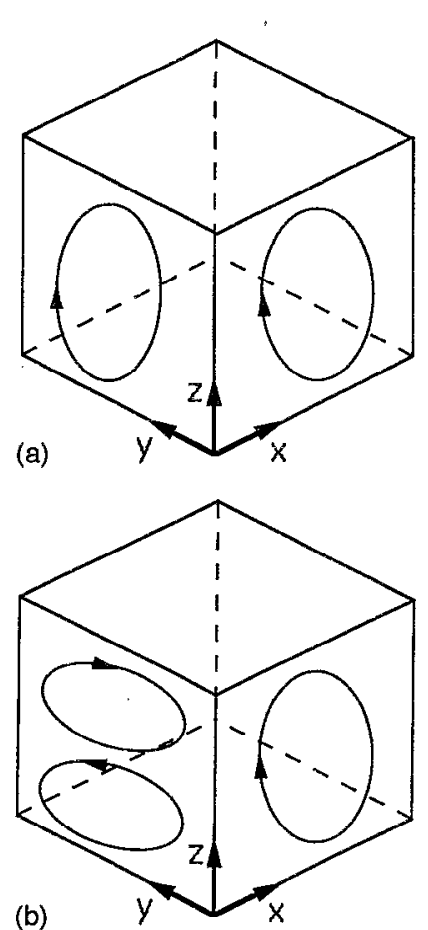

FIG. 1. Sketch of the flow fields: (a) integrable case, (b) nonintegrable case.

$$
\mathbf{U}_{1}(\mathbf{x})=U_{1}\left(-\sin (\pi x) \cos (\pi z) \mathbf{e}_{x}+\cos (\pi x) \sin (\pi z) \mathbf{e}_{z}\right),
$$

where $\mathbf{e}_{x}$ and $\mathbf{e}_{z}$ are unit vectors in the direction of the $x$ and $z$-coordinates respectively. Superimposing a second identical field at right angles to the first does not lead to any chaotic streamlines. Nevertheless, such a flow will be considered later as a prototypical nonchaotic, three-dimensional, example of convecting flow. To obtain chaotic behavior of the flow, it is necessary that the dependence in $z$ (or $x$ ) is different for the second recirculating flow. Thus we superpose the following field:

$$
\begin{aligned}
\mathbf{U}_{2}(\mathbf{x})= & U_{2}\left(-2 \sin (\pi y) \cos (2 \pi z) \mathbf{e}_{y}\right. \\
& \left.+\cos (\pi y) \sin (2 \pi z) \mathbf{e}_{z}\right) .
\end{aligned}
$$

Figure 2 shows the streamline patterns for both twodimensional velocity fields $\mathbf{U}_{1}$ and $\mathbf{U}_{2}$. As for any such flows, the chaotic properties depend crucially on the relative values of the amplitudes $U_{1}$ and $U_{2}$ of each contribution. Since we are interested in the efficiency of mixing, comparisons must be performed for comparable energy costs; for Stokes flows, the energy cost is directly related to the global viscous dissipation rate, defined as

$$
\Phi=\int_{\Omega} 2 \nu \mathbf{D}(\mathbf{U}) \cdot \mathbf{D}(\mathbf{U}) d^{3} \mathbf{x}
$$

where $\mathbf{D}(\mathbf{U})$ is the rate of straining tensor of the flow. Thus, requiring that $\Phi$ is constant leads to:

$$
U_{1}^{2}+\frac{25}{4} U_{2}^{2}=1 \text {. }
$$

From equation (2) and using the boundary conditions for $c(\mathbf{x}, t)$ and $\mathbf{U}(\mathbf{x})$, one can derive the evolution equation for the mean square gradient (see Appendix A): 


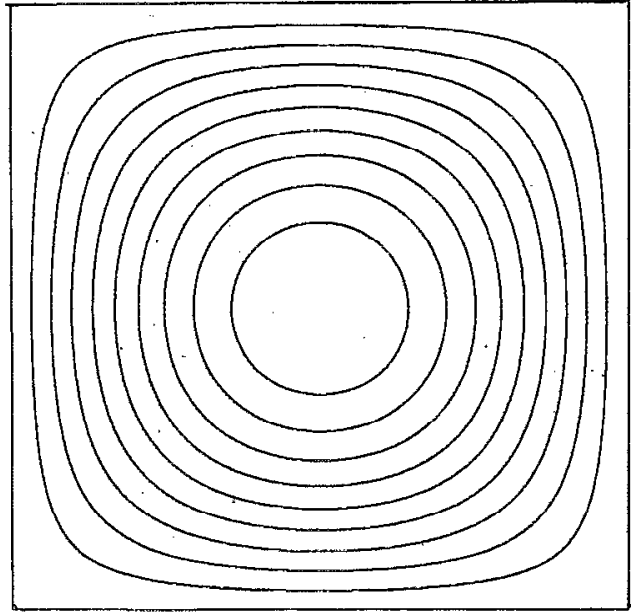

(a)

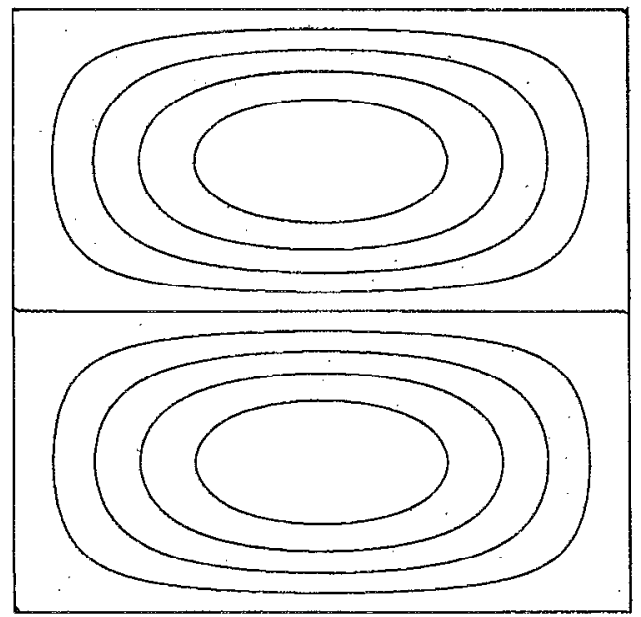

(b)

FIG. 2. Streamlines of the two-dimensional fields: (a) $\mathbf{U}_{1}$; (b) $\mathbf{U}_{2}$.

$$
\begin{aligned}
\frac{d}{d t} \int_{\Omega}|\nabla c|^{2} d^{3} \mathbf{x}= & -2 \int_{\Omega} \nabla c \cdot \mathbf{D}(\mathbf{U}) \cdot \nabla c d^{3} \mathbf{x} \\
& -\frac{2}{P e} \int_{\Omega}\left(\nabla^{2} c\right)^{2} d^{3} \mathbf{x} .
\end{aligned}
$$

Apparently, the production term for the mean square gradient is the action of this tensor on the gradient of the scalar field. Thus condition (13) also implies that different velocity fields have a priori the same ability to create large mean gradients.

Classical tools for characterizing the chaotic properties of streamlines induced by a flow field are Poincare sections and Lyapunov exponents (for detailed presentation, see, for instance, Guckenheimer and Holmes ${ }^{16}$ or Wiggins, ${ }^{17}$ and, in the context of Lagrangian chaos, Ottino ${ }^{1}$ ). Here, a section may be simply considered as a plane intersecting $\Omega$. In Fig. 3 we present Poincare sections of the streamlines for some typical values of the parameters $U_{1}$ and $U_{2}$ satisfying condition (13). For present purposes, it is sufficient to consider the section as the plane defined by $y=0.5$. These sections are computed numerically by integrating a single trajectory us- ing an explicit fourth order Runge-Kutta scheme in double precision arithmetic. In the particular case $U_{1}=0.5$, we also computed two specific trajectories chosen so that they each remain in the regular region. In each section, the number of points is about 10000 . Note that, to facilitate later comparisons (see section IV), we did not take into account the sign of the normal component of the velocity with which the streamline intersects the plane. On decreasing the value of $U_{1}$ from 1 (a case for which the streamlines are integrable), the chaotic region grows progressively larger and covers the entire domain when approximately $U_{1}=0.25$. On further decreasing the value of $U_{1}$, one observes that the chaotic region seems not to cover the domain uniformly. In fact, choosing the initial location of a particle in what appears to be an empty region in the present Poincare section reveals that the particle at first wanders about in this region for a while before escaping into the region shown to be densely covered by the present picture. At this stage, it is not clear to what extent the flow is ergodic in this case, since, due to numerical inaccuracy, it is not known if the particle must actually escape from the empty region appearing in the present figure or not. This phenomenon will have some consequences for the determination of Lyapunov exponents.

To provide more quantitative information on the chaotic behavior of the streamlines, a computational estimation of the Lyapunov exponents was performed. By linearizing the evolution equation of a fluid particle:

$$
\frac{d \mathbf{x}}{d t}=\mathbf{U}(\mathbf{x})
$$

one obtains the evolution equation of an infinitesimal vector $\varepsilon(t)$ of arbitrary orientation under the action of the flow field:

$$
\frac{d \varepsilon}{d t}=\varepsilon \cdot \nabla \mathbf{U}
$$

Classically, for most initial orientations and initial points $\mathbf{x}(0)$, the norm of the vector increases with time. If the streamlines form closed orbits or lie on a torus, it increases linearly with time. If the streamlines exhibit chaotic behavior, the growth of $\varepsilon(t)$ is of exponential type: the Lyapunov exponent is then:

$$
\lambda(\mathbf{x}(0), \boldsymbol{\varepsilon}(0))=\lim _{t \rightarrow \infty} \frac{1}{t} \ln \frac{\|\boldsymbol{\varepsilon}(t)\|}{\|\boldsymbol{\varepsilon}(0)\|}
$$

where $\|\cdots\|$ denotes some norm in $\mathbf{R}^{3}$. Such a limit is known to exist ${ }^{18}$ under certain smoothness assumptions on the velocity field. More precisely, the solution of the linear nonautonomous equation (16) may be obtained by:

$$
\varepsilon(t)=A^{t} \cdot \varepsilon(0)
$$

where $A^{t}$ is the linear operator which maps $\varepsilon(0)$ to $\varepsilon(t)$. There exists a triad of Lyapunov exponents which can be ordered: $\lambda_{1} \geqslant \lambda_{2} \geqslant \lambda_{3}$. These exponents are $a$ priori dependent on the initial location of the trajectory, $\mathbf{x}(0)$. Nevertheless, we expect that when chaos is global the trajectory eventually wanders everywhere in the domain so that taking the limit 


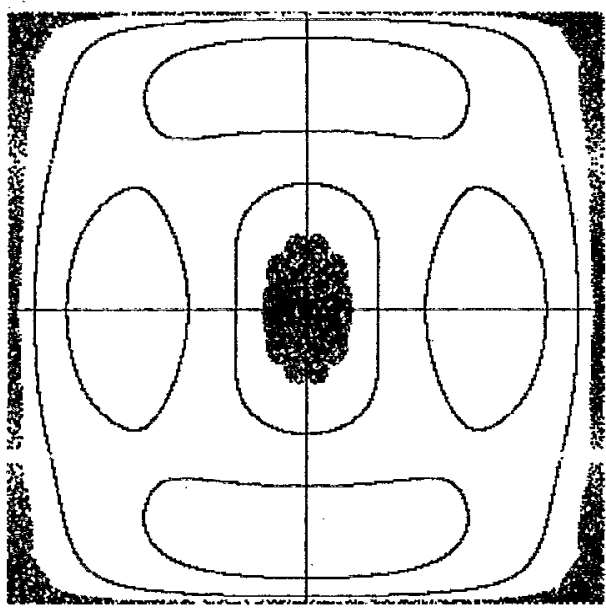

(a)

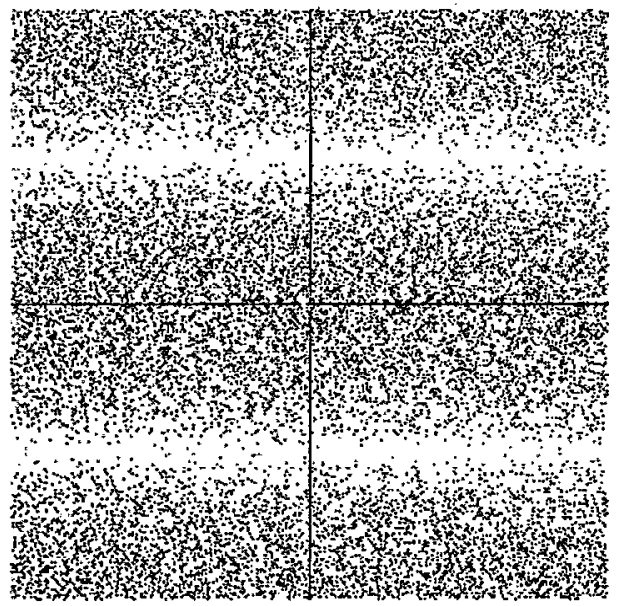

(b)

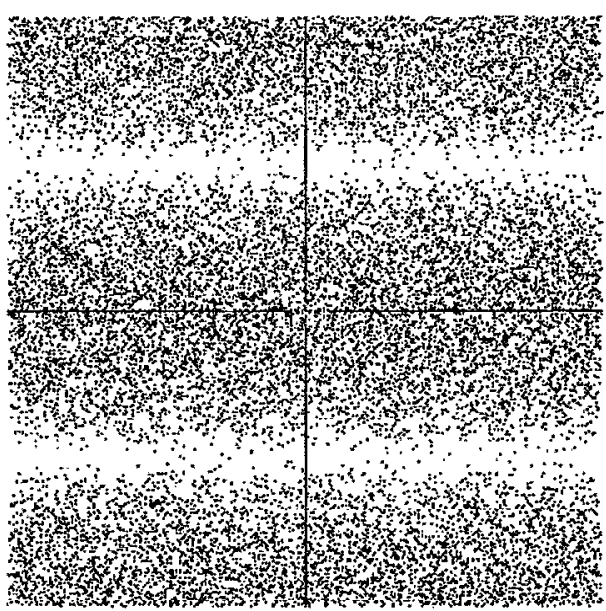

(c)

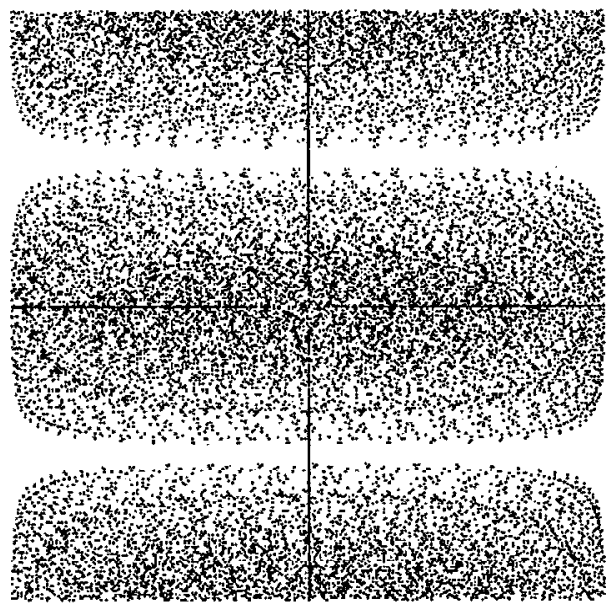

(d)

FIG. 3. Poincare sections of the streamlines generated by the flow field $\mathbf{U}_{1}+\mathbf{U}_{2}$. Section plane is $y=0.5$. Parameter values for $U_{1}$ and $U_{2}$ are respectively: (a) $0.5,0.346$; (b) $0.25,0.387$; (c) $0.15,0.395$; (d) $0.05,0.399$.

for $t \rightarrow \infty$ should lead to the same value for any initial point. Our computational results will partially confirm this last conjecture.

Some properties of the spectrum of Lyapunov exponents in the present case need to be emphasized and provide a check on the numerical computations. Since the flow field is divergence free, the trace of the gradient tensor is zero which implies that the sum of the three Lyapunov exponents is zero (the integration of the trace with time is simply the change of volume of a fluid particle). Moreover, the Lyapunov exponent in the direction of the streamline is zero for all streamlines except a set of zero measure-including, for instance, some of the particular streamlines of the boundary of the domain-since equation (15) is invariant in time: the distance between two points of the same streamline is bounded for all times. Consequently, for chaotic streamlines, we have $\lambda_{1}>0, \lambda_{2}=0$ and $\lambda_{3}=-\lambda_{1}$, so that the mean dispersion property of the flow may be estimated from only one statistical value, $\lambda_{1}$.

The relationship between the Lyapunov exponents and the mixing mechanism becomes clear from the equation for the gradient of the scalar field which can be derived from (2) (see Appendix A):

$$
\frac{d}{d t} \nabla c=-\nabla c \cdot \nabla \mathbf{U}^{T}+\frac{1}{P e} \nabla^{2} \nabla c .
$$

Clearly, if we ignore the diffusion term of equation (19), $\nabla c$ satisfies an equation similar to equation (16) (there is no influence for the transposition on the value of the Lyapunov exponents and the consequence of the opposite sign is essentially to exchange $\lambda_{1}$ with $\lambda_{3}$ and vice-versa). Thus, in a chaotic region, the Lyapunov exponents measure the mean rate at which the gradient of the scalar field is produced under the action of the flow field. In fact, this mechanism is limited by diffusive effects involving the second term of the right hand side of equation (19).

The computation of Lyapunov exponents was performed using the method proposed by Eckmann and Ruelle ${ }^{18}$ (see also Manneville ${ }^{19}$ ). We verified that the Lyapunov exponents are actually zero for both the two limiting regular cases $U_{1}=1$ and $U_{1}=0$ of the flow field, as well as for the three- 


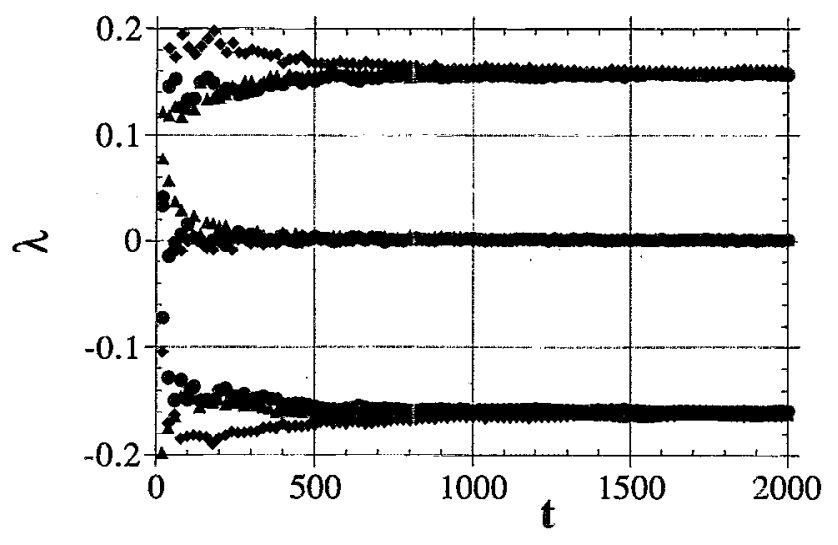

FIG. 4. Lyapunov exponents of the flow field $\mathbf{U}$ for $U_{1}=0.25$ and $U_{2}=0.387$ and three different trajectories corresponding to the following values of $\mathbf{x}(0):(\mathbf{\Lambda})(0.25,0.5,0.75) ;(0)(0.4,0.5,0.7) ;(\bullet)(0.1,0.5,0.1)$ respectively.

dimensional case of two identical recirculating flows at right angles. We also checked that, when both chaotic and regular regions are present, the Lyapunov exponent $\lambda_{1}$ effectively tends to a zero value for a trajectory lying inside the regular domain, and to a strictly positive for a trajectory inside the chaotic region. Since, in this case, the resulting value of the positive Lyapunov exponent is not typical of the whole flow, we next focus our attention on flows exhibiting global chaotic behavior in Poincaré sections. The numerical results for the Lyapunov exponents in the case $U_{1}=0.25$ are shown in Fig. 4 for three different locations of the initial point $x(0)$. As expected, for sufficiently long times, we obtain one positive and one negative value of the same magnitude, and a third one close to zero. It is also observed that, for this flow, the asymptotic values do not depend on the location of the initial point. Thus, in this case, the positive Lyapunov exponent seems to be a rather good statistical measurement of chaos for use in mixing time prediction. Also note that the asymptotic value is reached for nondimensional times of order 500 , which must be compared with the characteristic times for evolution of the scalar field given in the following section.

A limitation on the use of the characteristic exponent in the prediction of mixing times arises when trying to distinguish between different but nearby configurations. Indeed, the asymptotic values of Lyapunov exponent for $U_{1}=0.25$ and $U_{1}=0.22$ do not exhibit any noticeable difference: in contrast, we shall see in subsequent sections that the mixing properties of these two flows are clearly different. Another limitation appears when computing the Lyapunov exponent in the case $U_{1}=0.05$, as shown in Fig. 5. After transient effects, the exponents stabilize at different values depending on the location of $\mathbf{x}(0)$. This fact is related to a previous observation: some parts of the fluid domain are rarely visited by the trajectory. Depending on whether the initial point is taken inside or outside these regions, the computed values are different. However, if a much larger time of integration is used $(\approx 40000)$, the asymptotic values approach each other. This eventual agreement is clearly due to the fact that, after a while, the particle escapes from the rarely visited region. Consequently, it is not known whether this agreement is

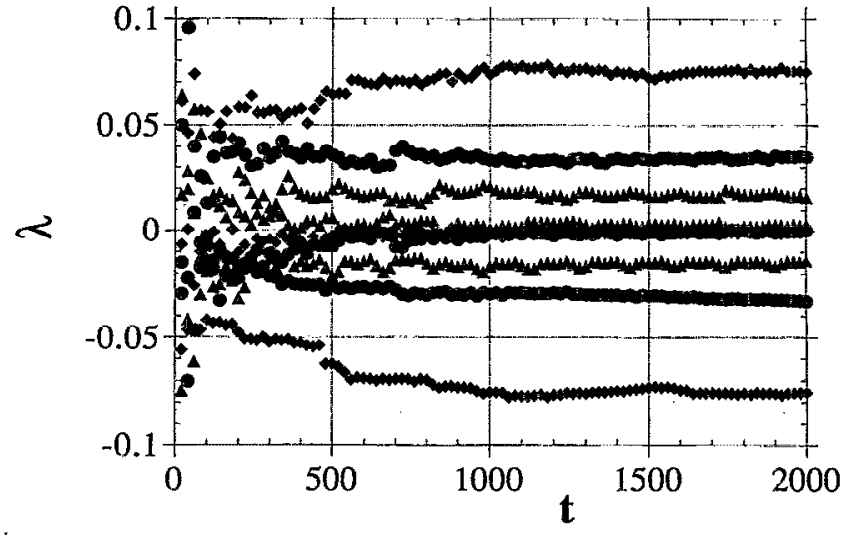

FIG. 5. Lyapunov exponents of the flow field $\mathbf{U}$ for $U_{1}=0.05$ and $U_{2}=0.399$ and three different trajectories corresponding to the following values of $\mathbf{x}(0):(\mathbf{A})(0.25,0.5,0.25) ;(\bullet)(0.4,0.5,0.7) ;(\bullet)(0.1,0.5,0.1)$ respectively.

physical or due to numerical inaccuracies in the trajectory computation. Moreover, the characteristic time required for this is roughly one hundred times larger than the characteristic time for evolution of the scalar field when $P e=10^{4}$ so, in the present context, the eventual agreement of values is of limited interest. The fact that the evaluation of the Lyapunov number at finite times for a given initial location of the trajectory can be different has already been mentioned in the literature, especially by Eckhardt and Yao, ${ }^{20}$ who proposed the use of local Lyapunov numbers. In section V, we will see how to relate one of the earlier limiting values (the one corresponding to initial location inside the rarely visited domain) for limited time (500) to the evolution of the scalar field.

\section{EVOLUTION OF THE SCALAR FIELD}

We turn now to the simulation of the scalar field subjected to convection-diffusion mechanisms. Most of the computations have been performed for a Péclet number of $10^{4}$, which is sufficiently large that the convection mechanism plays an important part in the mixing process, yet not so large as to preclude accurate simulation given the limited number of modes in each direction. The initial scalar fields is chosen to have random amplitudes of the first four modes in each direction, suitably scaled so that condition (5) is satisfied while all other modes have zero amplitude. This choice is consistent with the fact that, on the one hand, the problem is the mixing of a large length scale scalar field and, on the other hand, taking too specific an initial condition may lead to specific evolution. A check on the accuracy of the computation by increasing the number of modes has been performed in the two cases of partially and globally chaotic streamlines. When $U_{1}=0.5$ for which the flow exhibits a sizeable regular region, the evolution had converged for a number of modes in each direction $(N)$ greater than 24. When $U_{1}=0.25$ for which chaos appears global, some differences in the rate of decay are still observed for $N$ up to 48 (see Fig. 6 ) but it seems that the accuracy obtained for $N=40$ is sufficient for the present purpose and all calculations at 


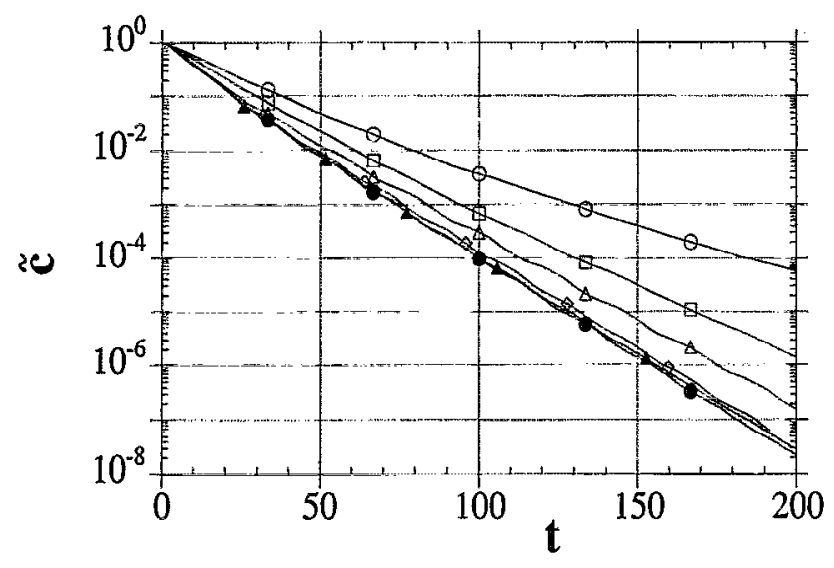

FIG. 6. Evolution of $\tilde{c}(t)$ for $U_{1}=0.25, U_{2}=0.387$ and for differents numbers of modes $N$ in each direction: $(O) 12 ;(\square) 16 ;(\triangle) 24 ;(\diamond) 32 ;(\bullet) 40$; (A) 48 .

$P e=10^{4}$ were performed using this number of modes in each direction. A similar check was performed by reducing the time step. Finally, the evolution obtained for different random values of the initial scalar field reveals that, as expected, the behavior remains the same indicating that mixing is essentially governed by the eigenvalue of highest real part.

Systematic computations were performed for the same initial scalar field and different values of the amplitude $U_{1}$ and $U_{2}$, see Fig. 7. The first and main conclusion which may be derived from Fig. 7 is that, disregarding transient effects for short times, there are actually optimal values of the amplitudes of the flow fields for which the mean decay of $\tilde{c}(t)$ is the fastest (recall that for all these values of the velocity amplitudes the energy cost remains constant). As shown in Fig. 8, the optimal value is approximately $U_{1}=0.25$, and corresponds, at least approximately, to the borderline between the presence and the absence of regular regions in the Poincaré sections. Similar computations for a Péclet number of $10^{3}$ lead to a similar optimal value of $U_{1}$. For larger values of the Péclet number it was not possible, owing to the computational cost, to perform such a parametric study, but the partial results we obtained (at $P e=10^{5}$ ) indicate that

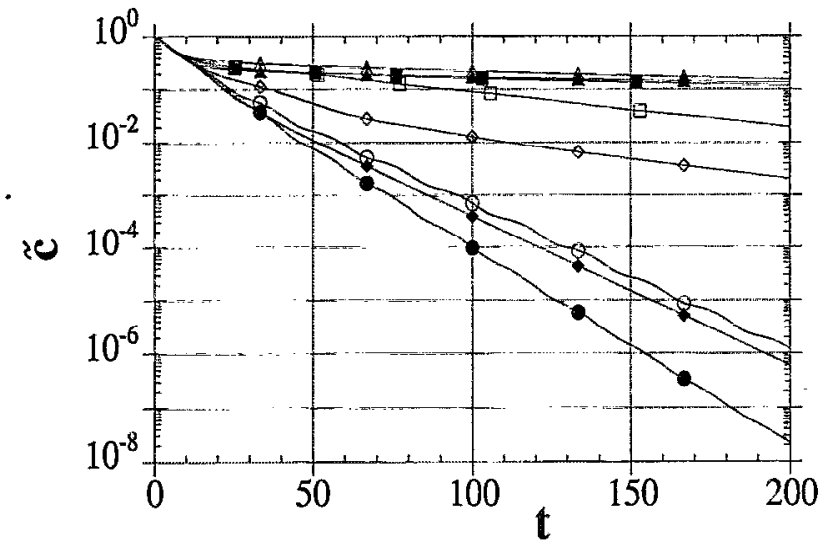

FIG. 7. Evolution of $\tilde{c}(t)$ for $U_{1}$ and $U_{2}$ being: $(\Delta) 0,0.4 ;(\Delta) 1,0 ;(\diamond)$

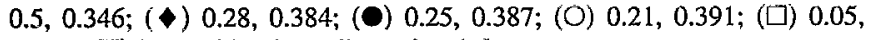
0.399. (ש) integrable, three-dimensional flow.

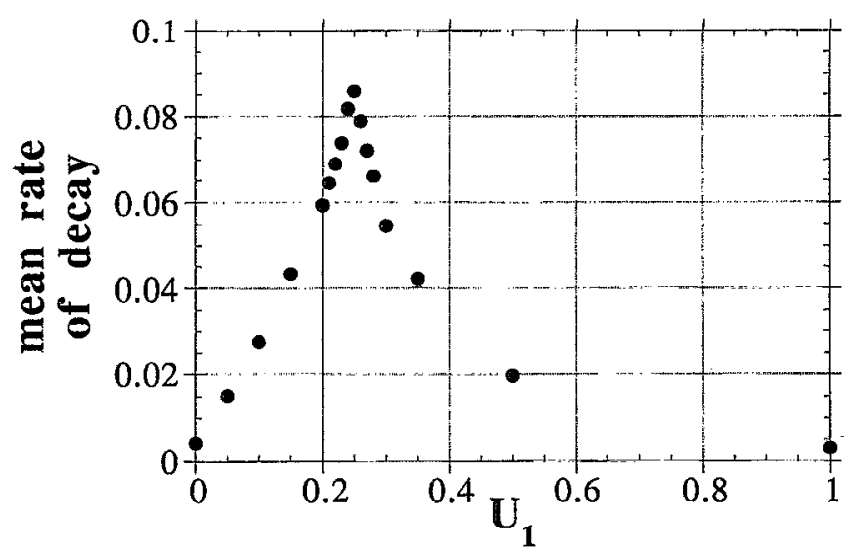

FIG. 8. Asymptotic mean rate of decay of $\tilde{c}(t)$ versus $U_{1}$.

similar optimal values should be expected. The existence of such optimal values is not surprising since for the two limiting integrable fields $\left(U_{1}=1\right.$ or $\left.U_{1}=0\right)$, which are twodimensional, the evolution is limited by purely diffusive effects in the third direction. Since the evolution for twodimensional flow may be considered as a special case because some of the eigenfunctions of the Laplace operator are invariant under the action the flow, we also considered the integrable, three-dimensional, flow. As may be seen in Fig. 7, the rate of decay is considerably lower than that obtained for the optimal case, and its asymptotic value is approximately the same as for two-dimensional flows. Note also in Fig. 8 that some chaotic flows are actually not very efficient, even when the chaos seems global (see the Poincare section for $U_{1}=0.15$ in Fig. 3). In fact, the efficiency decreases continuously when $U_{1}$ decreases below 0.25 . For very small $U_{1}$, global mixing is limited by the small remaining exchange between what appears to be an empty region in the Poincare section (Fig. 3) and the remainder of the domain.

Another interesting feature of Fig. 7 is that, for flows exhibiting regular regions, the evolution of $\tilde{c}(t)$, after transient effects, appears as a straight line in a log representation whereas modulations occur for global chaotic fields. In our case, il is particularly easy to obtain the instantaneous rate of decay, since, from equation (7):

$$
\frac{d \ln \tilde{c}}{d t}=-\frac{1}{P e} \frac{\int_{\Omega}|\nabla c|^{2} d^{3} \mathbf{x}}{\int_{\Omega} c^{2} d^{3} \mathbf{x}} .
$$

The evolution of the right hand side of this equation is plotted in Fig. 9. It appears that when sufficiently large regular regions are present, the exponential rate of decay tends to a time-independent value, reflecting the fact that the dominant eigenvalues are of real type. In this case, we show in Appendix B that there exists a limitating diffusive effect which involves only the geometry of the regular region of the flow, so that, when large regular regions are present, slow diffusive scales are involved. At the same time, we observe that the isovalue surfaces of the scalar field align asymptotically with the torus of the regular region. This phenomenon is clearly shown in Fig. 10, where we have plotted successive sections of the scalar field in the plane $y=0.5$, which was the plane 


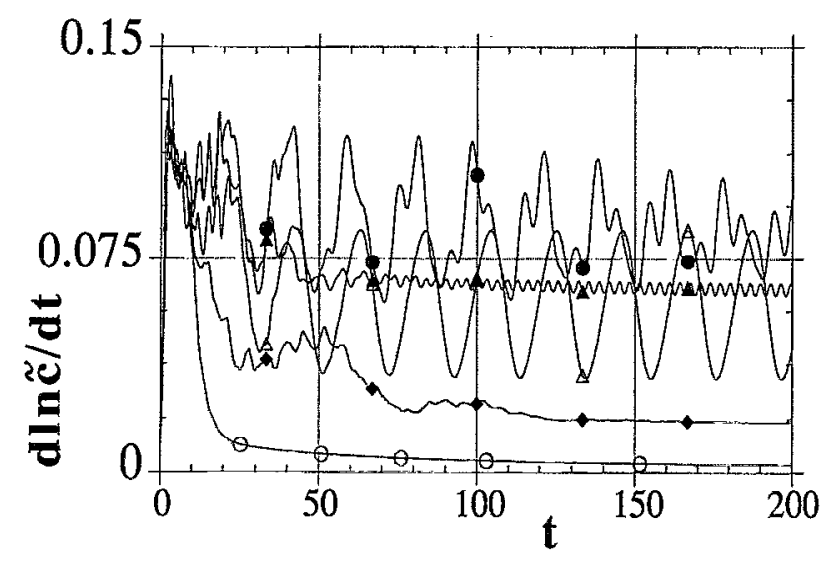

FIG. 9. Evolution of $d \ln (\tilde{c}) / d t$ for $U_{1}$ and $U_{2}$ being: (O) $0.5,0.346 ;(\triangle)$ $0.28,0.384:(\bullet) 0.25,0.387 ;(\Delta) 0.2,0.392$. (४) integrable, threedimensional flow. used for Poincare sections in Fig. 3. In these plots, in order to show the geometry of the isoscalar surfaces, the difference between two contours has been adjusted to take into account the mean decay of the scalar field. The initial field is as described earlier. For the partially chaotic flow, the scalar field is clearly tending to a time independent pattern: of course, some unsteady effects are still apparent since several eigenfunctions are contributing, but the main characteristics of the streamlines in the regular region are recovered. As expected, the asymptotic evolution of the scalar field is essentially related to the geometry of the regular region of the flow. Of course, as the size of the regular region decreases, the influence of this diffusive mechanism decreases too: the influence of the right hand side term in equation (B4) (see Appendix B) becomes more and more important which explains the appearance of small oscillations of the rate of decay for flows with small regular regions.

For globally chaotic flows, the numerical simulation re-
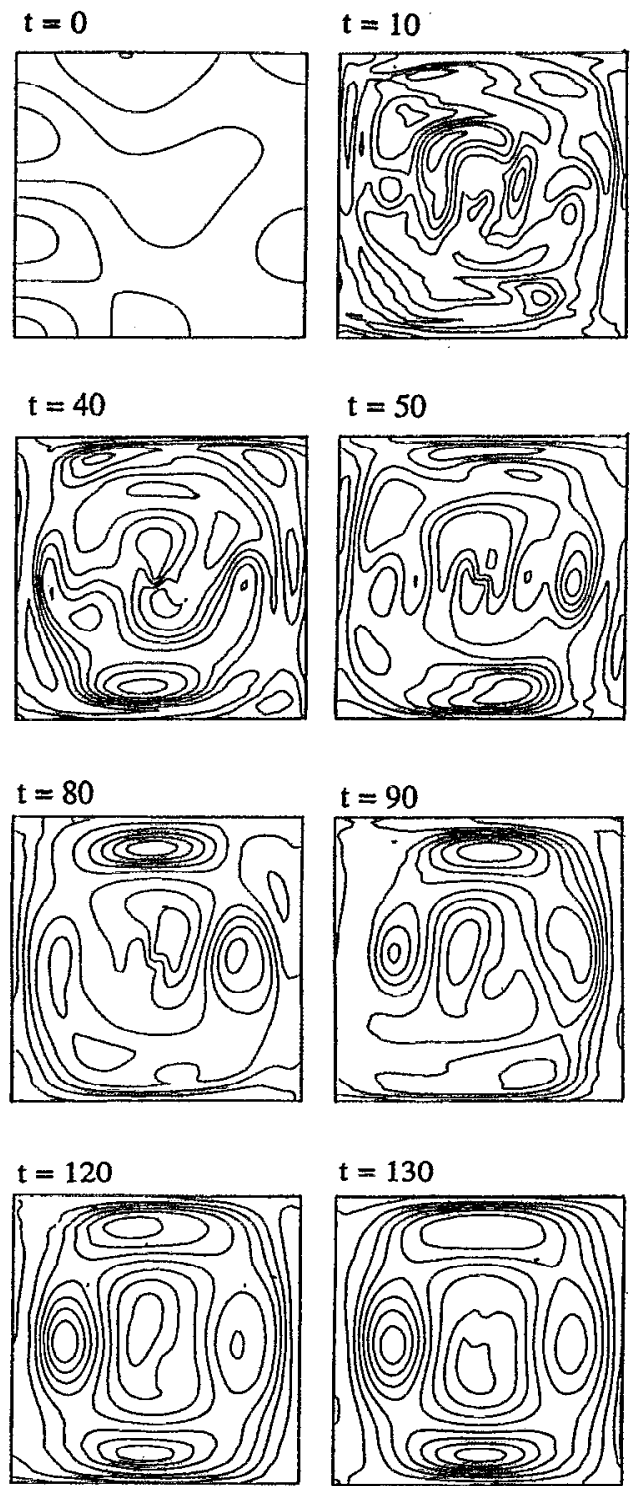

$t=130$

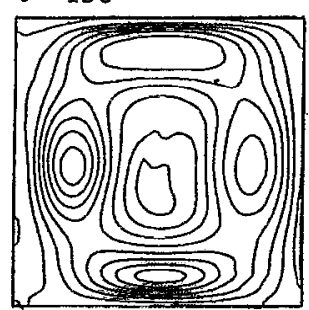

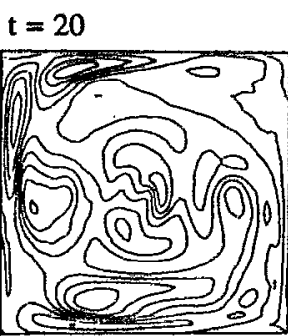
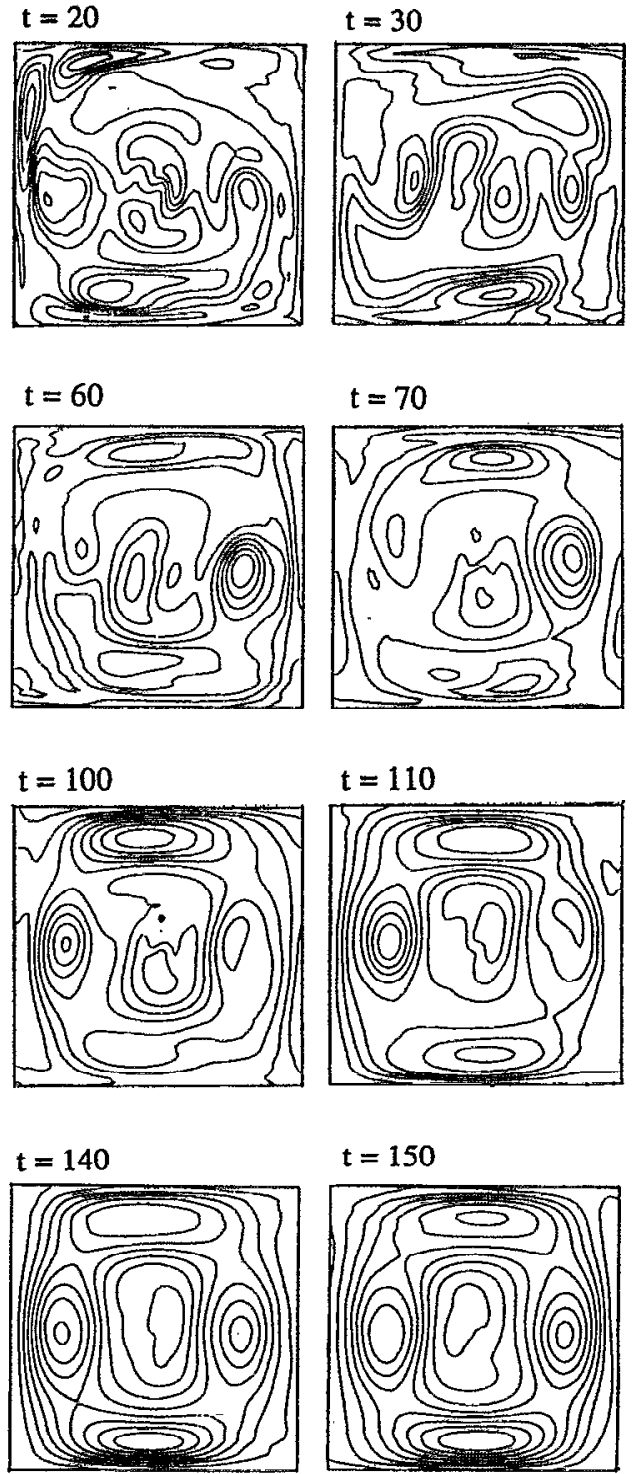

FIG. 10. Evolution of the scalar field for $U_{1}=0.5, U_{2}=0.346$. The plane is $y=0.5$. The difference between two neighbouring contours is made to decrease with time according to the mean decay of $c(x, t)$. 

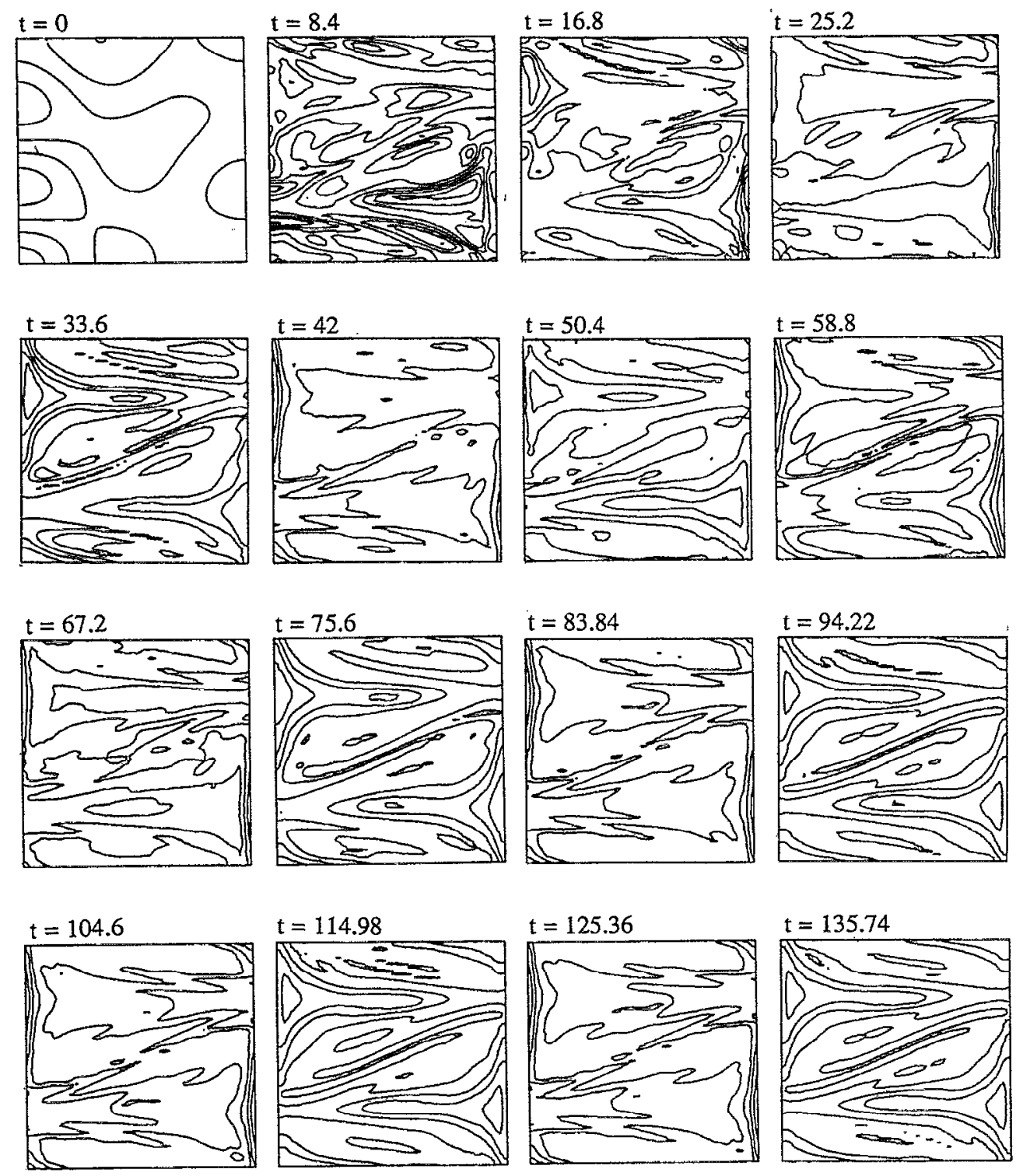

FIG. 11. Evolution of the scalar field for $U_{1}=0.20, U_{2}=0.392$. The plane is $y=0.5$. The difference between two neighbouring contours is made to decrease with time according to the mean decay of $c(x, t)$. The time interval between successive frame is adjusted according to the periodicity of the mean rate of decay of $c(\mathbf{x}, t)$.

veals that the exponential rate of decay oscillates indicating that some of the leading eigcnvalues are of complex type. More precisely, one can distinguish between two types of behavior. According to Fig. 9 , for $U_{1}=0.2$ the time evolution of the rate of decay becomes periodic after a transient effect. This is also clear from the evolution of the scalar field itself (Fig. 11). In this case, the time interval between each picture has been adjusted to illustrate the time periodicity of the rate of decay. Such a behavior is found for the entire range $U_{1} \leqslant 0.23$, where the frequency decreases for decreasing $U_{1}$. An analysis of the evolution of the rate of decay shows that essentially only one frequency is involved, the relative influence of the harmonics being only a few percent. This reflects the fact that, for most of the present flow fields exhibiting global chaos, the eigenvalue of the advection-diffusion operator of highest real part is well isolated from the other ones. However this is not the case for a small range of the parameter $\left(0.24 \leqslant U_{1} \leqslant 0.26\right.$, if we ignore the small oscillations obtained for values close to 0.26 ) in the vicinity of the optimal value: no periodic evolution of the rate of decay is observed for these flow fields (see Fig. 9 and Fig. 12). Several eigenvalues with incommensurate frequencies then have similar real parts. The sensitivity of this behavior on the number of modes was partially checked; increasing the number of modes to $N=48$ did not change the overall characteristics of the time evolution of the rate of decay. We also suspect that they are dependent on $P e$ : computations for a smaller Péclet number $\left(P c=10^{3}\right)$ gave only quasi-constant and periodic evolution.

In short, at $P e=10^{4}$ we obtained three different types of evolution of the rate of decay depending on the parameter $U_{1}$ and consequently on the topology of the streamlines: a quasiconstant rate for $U_{1}>0.26$, a time periodic evolution for $U_{1} \leqslant 0.23$, and an aperiodic evolution between these values. 

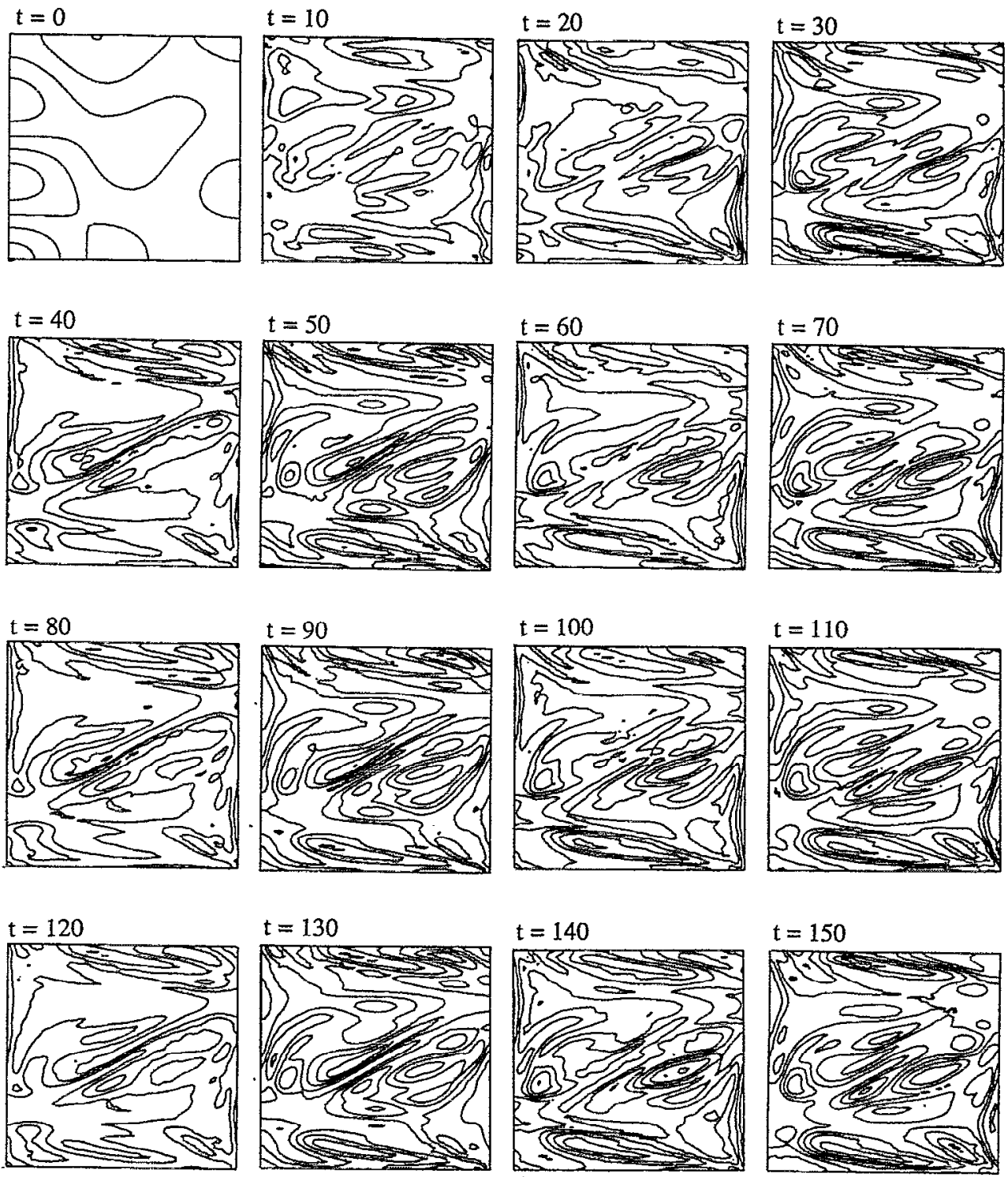

FIG. 12. Evolution of the scalar field for $U_{1}=0.25, U_{2}=0.387$. The plane is $y=0.5$. The difference between two neighbouring contours is made to decrease with time according to the mean decay of $c(\mathbf{x}, t)$.

The quasi-constant rate may be explained by the presence of regular regions, while the physical mechanism of the other types of behavior (for instance, the relationship between the observed frequencies and the properties of the flow field) remains an open question.

\section{RELATIONSHIP BETWEEN LYAPUNOV EXPONENTS AND TIME EVOLUTION OF THE SCALAR FIELD}

For globally chaotic flows, we attempted to relate the mean rate of decay obtained in section IV to the values of the Lyapunov exponents obtained in section III. For this purpose, we used the following heuristic approach, based on an idea of Aref and Jones. ${ }^{12}$ Let us denote a characteristic nondimensional length scale of the scalar field by $l$ : the characteristic nondimensional tine $\tau_{c}$ to reach the scale $l$ by advection, starting at scale 1 may be roughly estimated as:

$$
l \approx \exp \left(-\lambda_{1} \tau_{c}\right)
$$

where $\lambda_{1}$ is the positive characteristic Lyapunov number of the flow. The characteristic diffusion time $\tau_{d}$ of this scale is simply given by:

$$
\tau_{d} \approx \frac{l^{2} P e}{C(\Omega)}
$$

In the present case we have $C(\Omega)=\pi^{2}$. Since the decay of $\tilde{c}(t)$ is due to the existence of a diffusion mechanism [see equation (7)], we assume that the mean exponential rate of decay corresponds to the inverse of some diffusion time $\tau_{\star}$, i.e.:

$$
\tilde{c}(t) \sim \exp \left(-\frac{t}{\tau_{\star}}\right) .
$$




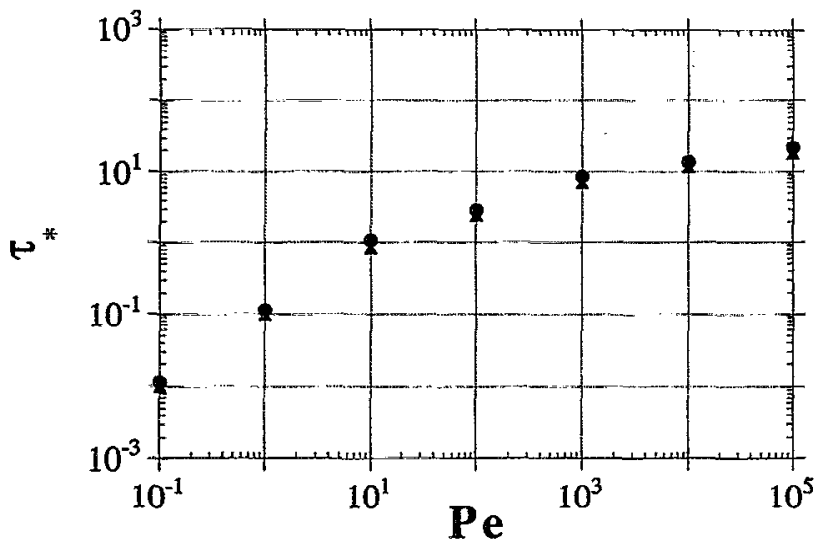

FIG. 13. Characteristic mixing time $\tau_{*}$ versus Péclet number. The values of the amplitudes of the flow field are: $U_{1}=0.25, U_{2}=0.387$. (-) numerical results, $(\boldsymbol{A})$ prediction using computed Lyapunov exponents.

We define $\tau_{\star}$ as the diffusion time of the length scale $l_{\star}$ so that $\tau_{c}=\tau_{d}$, i.e. when the rate of creation of $l_{\star}$ by advection is equal to its rate of dissipation by diffusion. Then $\tau_{\star}$ is solution of:

$$
\tau_{\star}=\frac{P e}{\pi^{2}} \exp \left(-2 \lambda_{1} \tau_{\star}\right)
$$

The time scale for decay, $\tau_{\star}$, exhibits a linear dependence at small Péclet numbers and a logarithmic one at large Péclet numbers. For integrable flows, this approach would lead to a dependence of $\tau_{\star}$ as $P e^{1 / 3}$ at large Péclet numbers, because the length scale then depends on the inverse of the characteristic convective time. A comparison of this heuristic prediction and the numerical results is shown in Fig. 13, which gives the mean value of $\tau_{\star}$ for Péclet numbers in the range 0.1 to $10^{5}$ for $U_{1}=0.25$ (the solution at $P e=10^{5}$ was obtained using 64 modes in each direction). In this case, the prediction agrees well with the numerical results. The quasilinear dependence for small Péclet number is recovered, while the asymptotic form at large Péclet number is also well described. Nevertheless, this apparently accurate agreement must be tempered by the fact that it is impossible to distinguish between the Lyapunov exponents of flows of similar but different amplitudes while their evolution can be significantly different, as shown in Fig. 7. Moreover, it must be recalled that, for this flow, the computed Lyapunov exponents are almost independent of the location of the initial point of the trajectory.

A thornier problem arises when we consider flows for which the computed Lyapunov exponents are dependent on the location of the initial point. In the case $U_{1}=0.05$, we obtained a minimum of the estimated Lyapunov exponent at finite time (that obtained for $t=500$ ) for a location of the initial point in the sparsely covered region. This corresponds to the smallest stretching in the fluid domain. We argue that the behavior in this sparsely visited region whose chaotic properties lead to the least rapid scalar exponential growth of gradients should produce the least rapid decay in $\tilde{c}(t)$. In

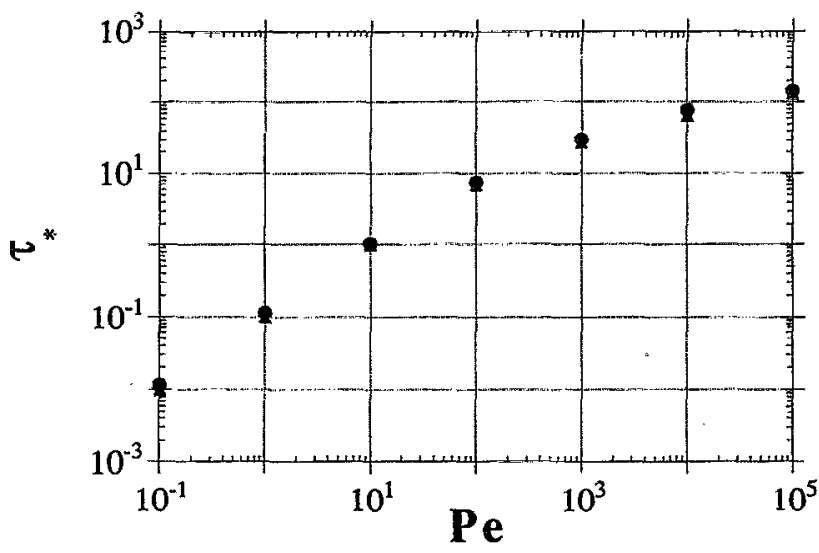

FIG. 14. Characteristic mixing time $\tau_{\star}$ versus Péclet number. The values of the amplitudes of the flow field are: $U_{1}=0.05, U_{2}=0.399$. (O) numerical results, (A) prediction using the lowest computed Lyapunov exponent.

attempting to recover the evolution of $\tau_{\star}$ by equation (24), we actually found the agreement when using this lowest value of $\lambda_{1}$ (see Fig. 14).

At this point, it should be observed that the preceding results could lead one to the erroneous conclusion that the determination of a single constant (the "smallest" Lyapunov exponent of the flow) is sufficient to describe global mixing. In fact, the two cases considered may be thought of as somewhat special: when $U_{1}=0.25$, a fluid particle wanders everywhere in the domain with an, at least approximately, uniform probability distribution, so that its asymptotic stretching is approximately the same whatever the location of the particle. At the other extreme $U_{1}=0.05$, there are two well-separated regions and the one with the smallest stretching efficiency essentially governs the mixing. In between these two cases, the situation is more complicated since finite time stretching is then dependent on the point of the domain we consider but not in the simple way of two separated regions. The asymptotic Lyapunov number for such intermediate flows does not give a satisfactory prediction of the mixing time and thus, for most cases, we would need a more local representation of finite time stretching. Moreover, the result would be dependent on the Péclet number considered.

\section{CONCLUSIONS}

The use of numerical simulation of the advectiondiffusion equation has permitted analysis of the global behavior under mixing of a scalar field by flow fields exhibiting chaotic streamlines. The evolution of the variance of the scalar field is well described by a modulated mean exponential decay. In particular, the mean rate of decay of the variance of the scalar field is nearby a constant when regular regions are present: this has been related to the fact that the isoscalar surfaces of the scalar field align asymptotically with the tori of the regular region. The efficiency of mixing is then essentially limited by a diffusive effect involving the geometrical properties (especially the size) of these regular regions. When chaos is global, oscillations of the mean rate of decay have been found: the periodicity of these oscillations depends both on the geometry of the streamlines and on the 
Péclet number. In this case, we have yet to find a satisfactory explanation of the results. We observe that the efficiency of mixing depends on the detailed properties of the streamlines inside the chaotic region: in particular, it has been shown that the presence of sparsely covered regions in the Poincare sections significantly affects the efficiency of mixing. In fact, the most important characteristic for efficient mixing is that the fluid particle wanders rapidly in the whole domain-i.e. does not remain for a long time in a particular region of the flow-even if the chaos appears global in the Poincaré sections. This observation may be important when considering more realistic flows, which usually do not exhibit homogeneous chaotic regions. It also affects the ability to predict the mixing time by a simple approach such as that using a global Lyapunov exponent.

\section{ACKNOWLEDGMENTS}

We wish to thank Professor D. Jeandel and Professor J.N. Gence. We especially thank Professor J.F. Scott for helpful discussions.

\section{APPENDIX A: DERIVATION OF THE NON-DIMENSIONAL GOVERNING EQUATIONS}

Let us consider a dimensional non-reacting scalar field $c^{*}\left(\mathbf{x}^{*}, t^{*}\right)$ satisfying the initial condition

$$
c^{*}\left(\mathbf{x}^{*}, 0\right)=c_{0}^{*}\left(\mathbf{x}^{*}\right) \text {. }
$$

Its evolution is governed by the equation:

$$
\frac{\partial c^{*}}{\partial t^{*}}+\mathbf{U}^{*} \cdot \nabla^{*} c^{*}=D \nabla^{* 2} c^{*}
$$

where $D$ is the molecular diffusivity of the scalar and $\mathbf{U}^{*}$ is the velocity field. Let $L$ be a characteristic length scale of the domain and $U$ a characteristic velocity scale. A characteristic scalar scale is defined as a deviation $\delta c$ with respect to a reference scalar $\bar{c}_{0}^{*}$ satisfying

$$
\bar{c}_{0}^{*}=\frac{1}{m e s(\Omega)} \int_{\Omega} c_{0}^{*} d^{3} \mathbf{x} .
$$

Thus, the non-dimensional scalar is defined as

$$
c=\frac{c^{*}-\bar{c}_{0}^{*}}{\delta c}
$$

Choosing the characteristic time scale as $L / U$, the nondimensional equation is

$$
\frac{\partial c}{\partial t}+\mathbf{U} \cdot \nabla c=\frac{D}{U L} \nabla^{2} c
$$

Introducing the Péclet number $\left(P e=\operatorname{ReS} C=\frac{\nu}{D} \frac{\nu L}{\nu}\right)$, we obtain finally

$$
\frac{\partial c}{\partial t}+\mathbf{U} \cdot \nabla c=\frac{1}{P e} \nabla^{2} c .
$$

We recall that $\mathbf{U}$ is a prescribed, divergence free, steady velocity field satisfying the boundary condition

$$
\mathbf{U} \cdot \mathbf{n}=0 \text { along } \Gamma
$$

and that the scalar field satisfies the initial condition

$$
c(\mathbf{x}, 0)=c_{0}(\mathbf{x})
$$

and the boundary condition

$$
\boldsymbol{\nabla} c \cdot \mathbf{n}=0 \text { along } \Gamma \text {. }
$$

Equation (5) is obtained by integrating equation (A6) over $\Omega$

$$
\int_{\Omega} \frac{\partial c}{\partial t} d^{3} \mathbf{x}+\int_{\Omega} \nabla \cdot(\mathbf{U} c) \dot{d}^{3} \mathbf{x}=\frac{1}{P e} \int_{\Omega} \nabla \cdot(\nabla c) d^{3} \mathbf{x} .
$$

And using the divergence formula

$$
\frac{d}{d t} \int_{\Omega} c d^{3} \mathbf{x}+\int_{\Gamma} c \mathbf{U} \cdot \mathbf{n} d \Gamma=\frac{1}{P e} \int_{\Gamma} \nabla c \cdot \mathbf{n} d \Gamma .
$$

Using conditions (A7) and (A9), one finds

$$
\frac{d}{d t} \int_{\Omega} c d^{3} \mathbf{x}=0
$$

So.

$$
\int_{\Omega} c d^{3} \mathbf{x}=\int_{\Omega} c_{0} d^{3} \mathbf{x}=0 .
$$

Equation (7) is obtained by multiplying equation (A6) by $c$ and integrating over $\Omega$, and so:

$$
\begin{aligned}
& \frac{1}{2} \frac{d}{d t} \int_{\Omega} c^{2} d^{3} \mathbf{x}+\int_{\Omega} \nabla \cdot\left(\frac{c^{2}}{2} \mathbf{U}\right) d^{3} \mathbf{x} \\
& \left.\quad=\frac{1}{P e} \int_{\Omega} \nabla \cdot(\nabla c) d^{3} \mathbf{x}-\int_{\Omega}(\nabla c)^{2} d^{3} \mathbf{x}\right) .
\end{aligned}
$$

Using the divergence formula,

$$
\begin{aligned}
& \frac{1}{2} \frac{d}{d t} \tilde{c}^{2}(t)+\int_{\Gamma} \frac{c^{2}}{2} \mathbf{U} \cdot \mathbf{n} d \Gamma \\
& \quad=\frac{1}{P e}\left(\int_{\Gamma} \nabla c \cdot \mathbf{n} d \Gamma-\int_{\Omega}(\nabla c)^{2} d^{3} \mathbf{x}\right) .
\end{aligned}
$$

Using conditions (A7) and (A9), one finds

$$
\frac{1}{2} \frac{d}{d t} \tilde{c}^{2}(t)=-\frac{1}{P e} \int_{\Omega}(\nabla c)^{2} d^{3} \mathbf{x} .
$$

The equation for the scalar gradient is easily deduced from equation (A6):

$$
\begin{aligned}
& \frac{\partial}{\partial t}\left(\frac{\partial c}{\partial x_{l}}\right)+\frac{\partial}{\partial x_{l}}\left(U_{j} \frac{\partial c}{\partial x_{j}}\right)=\frac{\partial}{\partial x_{l}}\left(\frac{\partial^{2} c}{\partial x_{j}^{2}}\right), \\
& \frac{\partial}{\partial t}\left(\frac{\partial c}{\partial x_{l}}\right)+\frac{\partial U_{j}}{\partial x_{l}} \frac{\partial c}{\partial x_{j}}+U_{j} \frac{\partial^{2} c}{\partial x_{j} \partial x_{l}}=\frac{\partial^{2}}{\partial x_{j}^{2}}\left(\frac{\partial c}{\partial x_{l}}\right) .
\end{aligned}
$$

Then,

$$
\begin{aligned}
& \frac{\partial}{\partial t} \boldsymbol{\nabla} c+\mathbf{U} \cdot \boldsymbol{\nabla}(\boldsymbol{\nabla} c)+\nabla c \cdot \nabla \mathbf{U}^{T}=\nabla^{2}(\nabla c), \\
& \frac{d}{d t} \nabla c=-\nabla c \cdot \nabla \mathbf{U}^{T}+\nabla^{2}(\nabla c) .
\end{aligned}
$$


Multiplying equation (A20) and integrating over $\Omega$, one obtains

$$
\begin{aligned}
\int_{\Omega} \frac{d}{d t} \frac{|\nabla c|^{2}}{2} d^{3} \mathbf{x}= & -\int_{\Omega} \nabla c \cdot \nabla \mathbf{U}^{T} \cdot \nabla c d^{3} \mathbf{x} \\
& +\frac{1}{P e} \int_{\Omega} \nabla c \cdot \nabla^{2}(\nabla c) d^{3} \mathbf{x}
\end{aligned}
$$

The left hand side of this equation can be written as

$$
\begin{aligned}
\int_{\Omega} \frac{d}{d t} & \frac{|\nabla c|^{2}}{2} d^{3} \mathbf{x} \\
\quad= & \frac{d}{d t} \int_{\Omega} \frac{|\nabla c|^{2}}{2} d^{3} \mathbf{x}+\int_{\Omega} \mathbf{U} \cdot \nabla \frac{|\nabla c|^{2}}{2} d^{3} \mathbf{x} \\
\quad & \frac{d}{d t} \int_{\Omega} \frac{|\nabla c|^{2}}{2} d^{3} \mathbf{x}+\int_{\Omega} \nabla \cdot\left(\mathbf{U} \frac{|\nabla c|^{2}}{2}\right) d^{3} \mathbf{x} \\
& =\frac{d}{d t} \int_{\Omega} \frac{|\nabla c|^{2}}{2} d^{3} \mathbf{x}+\int_{\Gamma} \frac{|\nabla c|^{2}}{2} \mathbf{U} \cdot \mathbf{n} d \Gamma .
\end{aligned}
$$

Using condition (A7), one finds

$$
\int_{\Omega} \frac{d}{d t} \frac{|\nabla c|^{2}}{2} d^{3} \mathbf{x}=\frac{d}{d t} \int_{\Omega} \frac{|\nabla c|^{2}}{2} d^{3} \mathbf{x}
$$

The first term of the right hand side of this equation can be written as

$$
\begin{aligned}
-\int_{\Omega} \nabla c \cdot \nabla \mathbf{U}^{T} \cdot \nabla c d^{3} \mathbf{x}= & -\int_{\Omega} \nabla c \cdot \mathbf{D}(\mathbf{U}) \cdot \nabla c d^{3} \mathbf{x} \\
& -\int_{\Omega} \nabla c \cdot \mathbf{\Omega}^{T}(\mathbf{U}) \cdot \nabla c d^{3} \mathbf{x}
\end{aligned}
$$

where $\mathbf{D}(\mathbf{U})$ is the rate of straining tensor of the flow and $\Omega^{T}(\mathrm{U})$ the rate of rotation tensor of the flow. Finally, using the fact that $\boldsymbol{\Omega}^{T}(\mathbf{U})$ is anti-symmetric, one finds

$$
-\int_{\Omega} \nabla c \cdot \nabla \mathbf{U}^{r} \cdot \nabla c d^{3} \mathbf{x}=-\int_{\Omega} \nabla c \cdot \mathbf{D}(\mathbf{U}) \cdot \nabla c d^{3} \mathbf{x} .
$$

The right hand side of this equation can be written as

$$
\begin{aligned}
\int_{\Omega} \nabla c \cdot \nabla^{2}(\nabla c) d^{3} \mathbf{x} \\
\quad=\int_{\Omega} \nabla\left(\nabla^{2} c \nabla c\right) d^{3} \mathbf{x}-\int_{\Omega}\left(\nabla^{2} c\right)^{2} d^{3} \mathbf{x}, \\
\quad=\int_{\Gamma} \nabla^{2} c \nabla c \cdot \mathbf{n} d \Gamma-\int_{\Omega}\left(\nabla^{2} c\right)^{2} d^{3} \mathbf{x} .
\end{aligned}
$$

Using condition (A9), one obtains finally

$$
\int_{\Omega} \nabla c \cdot \nabla^{2}(\nabla c) d^{3} \mathbf{x}=\int_{\Omega}\left(\nabla^{2} c\right)^{2} d^{3} \mathbf{x}
$$

Using both expressions in equation (A21), one finds

$$
\begin{aligned}
\frac{d}{d t} \int_{\Omega}|\nabla c|^{2} d^{3} \mathbf{x}= & -2 \int_{\Omega} \nabla c \cdot \mathbf{D}(\mathbf{U}) \cdot \nabla c d^{3} \mathbf{x} \\
& -\frac{2}{P e} \int_{\Omega}\left(\nabla^{2} c\right)^{2} d^{3} \mathbf{x} .
\end{aligned}
$$

\section{APPENDIX B: DERIVATION OF A DIFFUSION-LIKE EQUATION FOR FLOWS WITH REGULAR STREAMLINE REGIONS}

Let us denote the infinite set of eigenfunctions of the Laplace operator by $\omega_{1}(\mathbf{x}), \omega_{2}(\mathbf{x}), \cdots$ having negative eigenvalues $-\mu_{1}^{2},-\mu_{2}^{2}, \cdots$, ordered so that $\mu_{1}^{2} \leqslant \mu_{2}^{2} \leqslant \cdots$. Suitably normalized, they form a basis of orthonormal functions. If a regular region exists in the flow, there is some smooth function $\chi(\mathbf{x})$ with zero normal derivative on $\Gamma$ :

$$
\chi(\mathbf{x})=\sum_{i \geqslant 1} \alpha_{i} \omega_{i}(\mathbf{x}), \text { with } \sum_{i \geqslant 1} \alpha_{i}^{2}=1,
$$

satisfying:

$$
\mathbf{U} \cdot \nabla_{\chi}=0 \text { in } \Omega \text {. }
$$

It is then possible to construct, using a suitable algorithm such as Gram-Schmidt orthogonalization, a new basis of orthonormal functions containing $\chi(\mathbf{x})$ and an infinite sequence

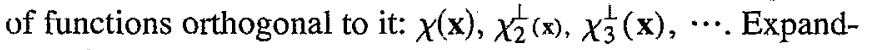
ing $c(\mathbf{x}, t)$ using this basis:

$$
c(\mathbf{x}, t)=C^{\| l}(t) \chi(\mathbf{x})+\sum_{j>1} C_{j}^{\lrcorner}(t) \chi_{j}^{1}(\mathbf{x}),
$$

inserting in equation (2), and using a Galerkin method, one finds the following equation for the evolution of $C^{\|}(t)$ :

$$
\begin{array}{r}
\frac{d}{d t} C^{\|}(t)+\frac{1}{P e}\left(\sum_{i \geqslant 1} \alpha_{i}^{2} \mu_{i}^{2}\right) C^{\|}(t) \\
=\frac{1}{P e} \sum_{j>1} C_{j}^{\perp}(t) \int_{\Omega} \nabla^{2} \chi_{j}^{\perp} \chi d^{3} \mathbf{x} .
\end{array}
$$

Any term involving $\mathbf{U}(\mathbf{x})$ has vanished because of condition (B2), incompressibility and the boundary conditions on the velocity field. Since equation (B4) is linear, the solution for $C^{\|}(t)$ is the sum of a general homogeneous solution and a particular solution. The homogeneous solution is easily found to have exponential decay at the rate: $P e^{-1} \Sigma_{i \geqslant 1} \alpha_{i}^{2} \mu_{i}^{2}$.

There is a particular function $\chi(\mathbf{x})$, satisfying condition (B2) and minimizing $\Sigma_{i \geqslant 1} \alpha_{i}^{2} \mu_{i}^{2}$. Such a function implies the existence of asymptotic diffusion effect which involves only the geometry of the regular region of the flow. For instance, when large regular regions are present, functions $\chi(\mathbf{x})$ exist for which the first coefficients $\alpha_{1}, \alpha_{2}, \ldots$ are dominant which implies that slow diffusive scales are involved. Note that $X(\mathbf{x})$ is not an eigenfunction of the problem, so that the right hand side of equation (B4) actually influences the evolution of $C^{\| l}$. What equation (B4) suggests is that large scale diffusion due to the presence of the regular region dominates the long time evolution of the scalar field. 
${ }^{1}$ J.M. Ottino, The Kinematic of Mixing: Stretching, Chaos and Transport (Cambridge University Press, Cambridge, 1989).

2 "Proceedings of an international symposium on fluids mechanics of stirring and mixing," Phys. Fluids A 3, 1089 (1991).

${ }^{3} \mathrm{H}$. Aref, "Stirring by chaotic advection," J. Fluid Mech. 143, 1 (1984).

${ }^{4} \mathrm{H}$. Aref and S. Balachandar, "Chaotic advection in a Stokes flow," Phys. Fluids 29, 3515 (1986).

${ }^{5} J$. Chaiken, R. Chevray, M. Tabor, and Q.M. Tan, "Experimental study of Lagrangian turbulence in a Stokes flow," Proc. R. Soc. London Ser. A 408, 165 (1986).

${ }^{6} \mathrm{~J}$. Chaiken, C.K. Chu, M. Tabor, and Q.M. Tan, "Lagrangian turbulence and spatial complexity in a Stokes flow," Phys. Fluids 30, 687 (1986).

${ }^{7}$ W.L. Chien, H. Rising, and J.M. Ottino, "Laminar mixing and chaotic mixing in several cavity flows," J. Fluid. Mech. 170, 419 (1986).

${ }^{8} \mathrm{M}$. Hénon, "Sur la topologie des lignes de courant dans un cas particulier" C.R. Acad. Sci. Paris 262, 312 (1966)

${ }^{9}$ T. Dombre, U. Frisch, J.M. Greene, M. Hénon, A. Mehr, and A.M. Soward, "Chaotic streamlines in the ABC flow," J. Fluid Mech. 167, 353 (1986).

${ }^{10} \mathrm{~K}$. Bajer and H.K. Moffatt, "On a class of steady confined Stokes flows with chaotic streamlines," J. Fluid Mech. 212, 337 (1990).
${ }^{11}$ T.J. Kaper and S. Wiggins, "An analytical study of transport in Stokes flows exhibiting large-scale in the eccentric journal bearing," J. Fluid Mech. 253, 211 (1993).

${ }^{12} \mathrm{H}$. Aref and S.W. Jones, "Enhanced separation of diffusing particles by chaotic advection," Phys. Fluids A 1, 470 (1989).

${ }^{13} \mathrm{~S}$.W. Jones, "The enhancement of mixing by chaotic advection," Phys. Fluids A 3, 1081 (1991).

${ }^{14}$ O.A. Ladyzenskaya and N.N. Ural'ceva, Equations Aux Dérivées Partielles de Type Elliptique (Dunod, Paris, 1968).

${ }^{15}$ C.W. Leong and J.M. Ottino, "Experiments on mixing due to chaotic advection in a cavity," J. Fluid Mech. 209, 463 (1989).

${ }^{16} \mathrm{~J}$. Guckenheimer and P. Holmes, Nonlinear Oscillations, Dynamical Systems, and Bifurcation of Vector Fietds (Springer-Verlag, New York, 1983).

${ }^{17}$ S. Wiggins, Global Bifuncations and Chaos (Springer-Verlag, New York, 1988).

${ }^{18}$ J.P. Eckmann and D. Ruelle, "Ergodic theory of chaos," Rev. Mod. Phys. 57,617 (1985).

${ }^{19}$ P. Manneville, Structures Dissipatives, Chaos et Turbulence (Aléa-Saclay, Gif-sur-Yvettc, 1991).

${ }^{20} \mathrm{~B}$. Eckardt and D. Yao, "Local Lyapunov exponents in chaotic systems," Physica D 65, 100 (1993). 